\title{
Early life stress leads to enhanced susceptibility to social stress in adult male $\mathrm{C} 57 \mathrm{BL} / 6$ mice
}

\author{
Kisaretova P.E. ${ }^{1,2 *}$, Reshetnikov V.V. ${ }^{1}$, Ershov N.I. ${ }^{1}$, Bondar N.P. ${ }^{1,2}$ \\ ${ }^{1}$ Institute of Cytology and Genetics, SB RAS, Novosibirsk, Russia \\ ${ }^{2}$ Novosibirsk State University, Novosibirsk, Russia \\ *e-mail:kisaretova@bionet.nsc.ru
}

Key words: early life stress, maternal separation, social defeat stress, H3K4me3, prefrontal cortex

Motivation and Aim: To this date human society collected a lot of empirical evidence on the capacity of early life surroundings to drive changes in cognition that persist through whole life of individual. In case of adverse experience, we see emotional and behavioral dysregulation. Failure to find appropriate treatment suggests our lack of understanding of processes responsible for these effects. Epigenetic mechanisms nowadays are the most promising thing to look into for the answers. We investigate the effects of adverse early life experience on susceptibility to social stress in adulthood on methylome of prefrontal cortex in male C57BL/6 mice.

Methods and Algorithms: Animals were separated into three groups: subjected to chronic social defeat stress (SS); prolonged maternal separation during early life period in combination with social stress in adulthood (MS + SS); normal rearing condition (NC). Statistical significance of behavioral tests was examined by one-way ANOVA. Prefrontal cortex tissue was used for mRNA and DNA extraction. Active promoter enrichment of DNA was accomplished using Native ChIP assay with H3K4me3 antibodies. ChIPseq and RNA-seq were carried out on Illumina HiSeq4000 platform, using 4 animals per group. Differential expression analysis was performed by DESeq2 package on R. Results with $p$-value $<0.01$ and fold change $>1.25$ were considered significant.

Results: Anxiety levels were increased in SS + MS group compared to NC control group $(p<0.05)$. Social behavior was significantly reduced in SS $(p<0.01)$ and MS + SS $(p<0.001)$ groups compared to NC group. Using RNAseq analysis1 8 genes were found to be differentially upregulated simultaneously in SS and SS + MS groups. The most promising of them were chosen for further analysis with qPCR using extended animal groups. Homer1a, Bdnf, Npas2 confirmed to be differentially expressed. ChIPseq analysis resulted in identification of 135 differentially methylated regions in both SS and $\mathrm{SS}+\mathrm{MS}$ groups compared to NC.

Conclusion: Early postnatal stress leads to enhanced outcomes of social stress in male C57BL/6 mice compared to group of animals subjected only to social stress. Genes found to be differentially expressed in experimental groups compared to control are associated with immune processes such as antigen presentation and T-cell mediated cytotoxicity. More detailed investigation of epigenetic profile is necessary for understanding molecular processes underlying susceptible or resilient phenotype enouncement.

Acknowledgements: Supported by the RFBR (18-34-00603). 